\title{
DEVELOPMENT OF DELIVERY OF SERVICES FROM OCEAN OBSERVING SYSTEMS - AN OPPORTUNITY TO PROMOTE COMMON APPROPACHES FOR A GLOBAL OCEAN OBSERVING SYSTEM
}

\author{
Harvey E. Seim ${ }^{(1)}$, Hans Dahlin ${ }^{(2)}$, Gary Meyers ${ }^{(3)}$, Rebecca Shuford ${ }^{(4)}$, Roger Proctor ${ }^{(5)}$ \\ ${ }^{(1)}$ University of North Carolina at Chapel Hill, 340 Chapman Hall, Chapel Hill, NC 27599 USA, \\ Email: hseim@email.unc.edu \\ ${ }^{(2)}$ EuroGOOS (European Global Ocean Observing System) Office, SMHI (Swedish Meteorological and Hydrological \\ Institute), SE-601 76 Norrköping, Sweden, Email: hans.dahlin@smhi.se \\ ${ }^{(3)}$ Integrated Marine Observing System, University of Tasmania, Private Bag 110, Hobart TAS 7001 Australia, \\ Email: Gary.Meyers@csiro.au \\ ${ }^{(4)}$ Marine Ecosystems Division, NOAA (National Oceanic and Atmospheric Administration) Fisheries Office of Science \\ and Technology, Silver Spring, MD 20910 USA, Email: Rebecca.Shuford@noaa.gov \\ ${ }^{(5)}$ IMOS (Integrated Marine Observing System) eMarine Information Infrastructure University of Tasmania, \\ Private Bag 21, Hobart, TAS 7001, Australia, Email: Roger.Proctor@utas.edu.au
}

\begin{abstract}
In the last decade, there has been tremendous development of regional ocean observing systems (ROOS) that provide information on ocean conditions immediately adjacent to the world's population centers. As these systems mature, it is vital to ensure that the ability to deliver societal services is adequately supported by the systems. This paper reviews developments in several areas that influence that ability. The first are the governance and organizational structures that have been created to guide the implementation of three ROOSs - in Europe, the United States, and Australia. The degree to which they provide a mechanism for engaging a broad range of service providers and addressing a variety of research issues is examined to identify similarities and differences in approaches. As ocean observing systems have matured, it has been recognized that to simultaneously engage and support a range of societal service providers, careful attention to regional-scale information management practices is needed, for unlike the governance and organizational structures that permit tailoring to fit regional needs, information management practices must be interoperable to accommodate interactions of providers on local to global scales. The pros and cons of different approaches to ROOS development, including observing, model and information management components being adopted by these and other regional systems are explored. There is an opportunity in the coming decade to promote development of new, sustained observations and an information services interface that unites the global, regional and coastal systems to support a broad range of societal service providers in a fairly seamless fashion. The need for integration from global to coastal services will become more urgent as communities face the need to adapt to climate change in the ocean. Data assimilation, reanalysis and prediction models provide a critical link between global observing and applications at a regional or local scale.
\end{abstract}

\section{INTRODUCTION}

In the twenty years since the Global Ocean Observing System, or GOOS (http://www.ioc-goos.org/) began one of the more difficult and slowly-evolving aspects of the program has been the design and development of regional alliances, governing bodies that are themselves comprised of a series of Regional Ocean Observing Systems (ROOS). Because they are envisioned as providing a connection to a broad cross-section of user communities, regional alliances necessarily must evolve a governance system that gives user communities a voice in prioritizing the activities of the ROOS. The user communities must also help define the services they desire, which in turn places requirements on the information management systems the ROOS must support. In this community white paper, we explore the development and status of three different regional alliances, seeking to document what has influenced the governance systems and the information management systems they have created to provide information and services to their user communities. Though the review is not comprehensive because it does not include all the regional organization effects within GOOS, the three regional alliances reviewed present a spectrum of approaches and convey the challenges faced in developing system compatibility on a global scale. The paper assesses features they share, and a few possible common approaches that may provide a shared framework for GOOS as it moves into its third decade.

\section{BACKGROUND}

The three regional alliances that are represented and described here are EuroGOOS (European Global Ocean Observing System) (which incorporates multiple ROOS), the Integrated Ocean Observing System, or IOOS (the U.S. contribution to GOOS, defined as a ROOS), and the Integrated Marine Observing System (the Australian National System). 


\subsection{Governance and Funding}

\subsubsection{EuroGOOS}

No single European country can monitor and predict all the sea areas, which influence its own economic, environmental and social conditions. Nor can a single country make a significant contribution to the global structure and implementation of the Global Ocean Observing System (GOOS).

EuroGOOS (http://www.eurogoos.org/) is an Association of Agencies, founded in 1994, to further the goals of GOOS, and in particular the development of Operational Oceanography in the European Sea areas and adjacent oceans. At its inauguration in 1994, EuroGOOS was established as an informal Association without subscription fee and members signed a Memorandum of Understanding (The EuroGOOS MoU). In 1999, the decision was taken to revise the constitution to that of a legal agreement (http://www.eurogoos.org/documents/eurogoos/downloa ds/eg99_18eurogoosagreement2009.pdf) without incorporation, and including a membership subscription. EuroGOOS now has 35 Members in 19 European countries.

The EuroGOOS task is to co-ordinate operational oceanography (i.e., the activity of systematic and long-term routine measurements of the seas and oceans and atmosphere, and their rapid interpretation and dissemination, see http://www.eurogoos.org/index.php?mainid=2\&subid=4) in 19 sovereign nations, and in addition, to promote and co-ordinate operational oceanography at the European level (27 EU-countries plus Iceland, Norway, Russia, Croatia, Ukraine, Georgia, Turkey). Several of these nations have IOOS-like (see below) systems run by public bodies and mainly with sustained funding. The national scale is the basic funding scale of operations. The European scale is an important (but far from the only one) scale for funding research and development, and for political initiatives. The regional (subregional) scale is where we can improve cost efficiency. It is also the scale of the European ROOSs. Currently there are five ROOSs, these being ARCTIC ROOS, BOOS (Baltic Operational Oceanographic System), NOOS (Northwest Shelf Operational Oceanographic System), IBIROOS (Iberian-Biscay-Irish ROOS) and MOON (Mediterranean Operational Oceanography Network). The main task of the ROOSs is to support national services and activities, especially if these include national obligations to European Union (EU) or intergovernmental conventions / agreements e.g. GOOS. In addition to this, by utilising the ROOSs, we are creating pan-European services to support the EU member states. That work is related to the EU Marine Strategy

(http://ec.europa.eu/environment/water/marine/index_en .htm), the Maritime Policy, and the Global Monitoring for Environment and Security (GMES) Programme (http://www.gmes.info).

EuroGOOS is established with full recognition of the importance of existing systems in research and operational oceanography in Europe at national and European scales.

Members of EuroGOOS co-operate to establish a concerted European approach to the following:

- Identifying European priorities for operational oceanography, promoting the development of the scientific, technology and computer systems for operational oceanography, and its implementation, assessing the economic and social benefits from operational oceanography.

- Contributing to international planning and implementation of GOOS and promoting it at the national, European and global level. EuroGOOS provides information regularly to the IOC-WMOUNEP (Intergovernmental Oceanographic Commission-World Meteorological OrganizationUnited Nations Environment Programme) Committee for GOOS (I-GOOS), and to the GOOS Steering Committee (GSC).

EuroGOOS activities are designed to collaborate with and maximise the benefits from existing activities in operational oceanography, promoting the integration of these activities within the framework of GOOS. Members of EuroGOOS collaborate and support the following groups of activities:

- Advancing European operational oceanography in GOOS

- Promoting development of European regional and local operational oceanography, taking into account the Modules of GOOS for the Coastal Zone, Health of the Ocean, Living Marine Resources, Climate, and Ocean Services.

- Promoting development of common European operational data procedures and services, including data quality control and data management for operational oceanography.

- Promoting research and pre-operational research that will solve problems relating to operational oceanography.

- Promoting pilot studies in GOOS operations, local, regional, or global.

- Promoting development of common European operational oceanographic services and products of maximum value to European Governments and Agencies, furtherance of European industries and service companies, and the protection of the environment and health in the European coastal and shelf seas. 
Strategy and Activities of EuroGOOS are overseen by a Director who reports to the EuroGOOS Board, which is composed of a Chairman and practicing scientists from subscribing members. EuroGOOS projects are developed through its four Working Groups, the Technology Plan Working Group (TPWG), the Science Advisory Working Group (SAWG), the EuroGOOS Product Working Group (EPWG) and the Data Management, Exchange and Quality Working Group (DATA-MEQ WG). These WGs are led by a member of the Board and enlist expertise from all members of EuroGOOS and the wider scientific community. International linkages and alliances are frequently sought, e.g. the linkage between ACT (the Alliance for Coastal Technologies) and EuroACT. In recent times EuroGOOS has had significant input into the development of projects developing Operational Oceanography, for example EuroGOOS project SEPRISE (Sustained, Efficient Production of Required Information Services) which demonstrated the viability of establishing a real-time reporting system for in-situ observations (http://www.seprise.eu), the EU co-funded MERSEA (Marine EnviRonment and Security for the European Area) which demonstrated an EU capability to develop global and regional operational observing and modelling systems (http://www.mersea.eu.org), ECOOP (European Conference on Object-Orient Programming) which is raising the standard of EU coastal observing and model forecasting systems (http://www.ecoop.org), and through the development of the GMES (Global Monitoring for Environment and Security) Marine Core Services (http://www.gmes.info/) which has the objective to streamline consistent European capacities for forecasting, monitoring and reporting on the ocean state, an to foster derived applications on specific environmental and safety issues, for both the global ocean and the regional European seas, which will be realised through the recently started MyOcean project (http://www.myocean.eu.org).

The final governance structure for regional and panEuropean activities is still under development, and has to be agreed between EU and the Members States. It is expected to be implemented before 2014 .

\subsubsection{U.S. GOOS, IOOS}

The U.S. contribution to GOOS (i.e. U.S. GOOS, the regional alliance, in GOOS parlance) is the Integrated Ocean Observing System (IOOS) (http://www.ioos.gov/). IOOS is a multidisciplinary system designed to enhance the U.S. ability to collect, deliver, and use ocean information. The goal is to provide continuous data on U.S. open oceans, coastal waters, and Great Lakes in the formats, rates, and scales required by scientists, managers, businesses, governments, and the public to support research and inform decision-making. IOOS is defined as having a coastal and a global component, the latter focusing on open ocean and international partner observing programs. For this document, we are concerned with the coastal element, which is itself comprised of two components, one federal and one non-federal. There are as many as 17 U.S. Federal agencies that participate in IOOS. Coordination of the contributions of these 17 agencies is presently achieved through the White House Council on Environmental Quality: Committee on Ocean Policy (CEQ:COP) (http://ocean.ceq.gov/). The non-federal partners are organized into 11 Regional Associations (RAs), each having geographic coverage over a portion of U.S. territorial waters. The National Oceanic and Atmospheric Administration (NOAA) has been designated as the lead federal agency for coordination and implementation across partners. Following is an overview of the history of events that resulted in IOOS and the evolution in the governance structure to its present state (see [1] for further details).

The first formal step towards establishing U.S. GOOS was congressional legislation in 1997 that established the National Oceanographic Partnership Program (NOPP), a federal program led by an interagency council that represents the largest U.S. federal agencies that have ocean policy as a significant portion of their organizational mandate. In 1998, the NOPP formed the U.S. GOOS Steering Committee (USGSC), a nongovernmental body with members from industry, academia, federal and state government and nongovernmental organizations. Members of the USGSC authored the documents that provided the initial description of the U.S. regional alliance, suggesting that it serve to integrate information on the oceans from many diverse and disparate sources (government and privately operated) within the nation's exclusive economic zone [2] and [3]. These initial visions also promoted a hierarchical organizational structure in which the non-federal components of the observing system would be organized into roughly 10 regional (from the national perspective) systems, which in turn would be composed of 10 local, or sub-regional, systems. The intent was to keep the number of institutions that would require coordination at a given level to a manageable size.

In 2000, the NOPP established Ocean.US, a federal interagency planning office to better define the nation's ocean observing system and how it should function. Over the next 8 years Ocean.US led the development of a series of documents that defined the overall goals, design, implementation and development of IOOS [4, 5, 6 and 7], the character of the regional components and a variety of supporting information (see www.ocean.us/oceanus_publications) which advanced the conceptual reality of IOOS. Importantly, IOOS was envisioned as having 3 main subsystems (observing, modeling and analysis, and data management and communications or DMAC) that together deliver 
information necessary to address societal and scientific needs, creating a "user-driven" system. The seven societal goals of IOOS are:

- Improve predictions of climate change and weather and their effects on coastal communities and the nation;

- Improve the safety and efficiency of maritime operations;

- Allow more effective mitigation of the effects of natural hazards;

- Improve national and homeland security;

- Reduce public health risks;

- Allow more effective protection and restoration of healthy coastal ecosystems; and

- Enable the sustained use of ocean and coastal resources.

The other significant influence on the governance of IOOS was the report of U.S. Commission on Ocean Policy

(http://oceancommission.gov/documents/full_color_rpt/ welcome.html), delivered to Congress in 2004. The Bush administration response, the Ocean Action Plan (http://ocean.ceq.gov/actionplan.pdf), was released in December 2004. It established a larger and more complex interagency governance, led by the Council on Environmental Quality (CEQ) of the White House. The recently-passed congressional legislation authorizing IOOS ("The Integrated Coastal and Ocean Observing Act of 2009", referred to below as the Oceans Act; http://www.govtrack.us/congress/bill.xpd?bill=h111367) will re-define the interagency agreements by reinstating the governing body of the NOPP as responsible for the policy and coordination oversight of IOOS. Hence, the organizational structure of IOOS is not yet finalized but is expected to be so by 2011 .

Though IOOS is a large partnership endeavour, NOAA has been formally identified as the lead federal agency of the effort. In 2007, NOAA established the NOAA IOOS Program Office to coordinate NOAA's relevant observing activities and to provide a consistent management function within the federal government. Its role has become yet more important since the closure of the Ocean.US office in September 2008. The NOAA IOOS Program Office, under the guidance of the CEQ:COP, thus serves as the day-to-day coordinating body for both federal and non-federal components of IOOS implementation in the U.S.

Although NOAA is the lead agency for coordination of the IOOS partnership, including the RAs, the RAs themselves are responsible for defining and implementing their own regional level governance structures. To adequately capture and respond to local constituent needs, the regional components of IOOS have sought to engage a broad range of participants in IOOS, including state and local government, industry, academic institutions, regional management councils and non-governmental organizations. The institutional membership of the RAs is typically $25-50$, and therefore a reasonably complicated form of governance is needed to define priorities for these organizations. To meet their unique requirements, the eleven existing regional associations have developed a variety of different approaches to their governance but all are operated as consortia, some under Memorandums of Understanding (MOUs), while others have incorporated as not-for-profit organizations. The RAs have also created an overarching governing body - the National Federation of Regional Associations (NFRA; http://www.usnfra.org/) to coordinate across the 11 RAs. NFRA represents the RAs at the federal level and coordinates the development of ocean observing systems in their region according to IOOS design principles. Each member RA appoints two representatives to serve on the Board of Directors, and each RA has one vote.

Funding for elements of IOOS began as early as 2000 but did not become a formal part of the annual federal budget process until 2007. At present, US\$25-35M in annual funding for the coastal component of IOOS exists through NOAA. A portion of these funds goes toward support of that agency's efforts in supporting IOOS, and through a cooperative agreements competitive process the majority to base funding for the 11 regional associations. Although it was a significant success to have IOOS provided for in the federal budget, present levels of funding are not sufficient to support operations and maintenance at required levels. With the signing of the Oceans Act, providing Congressional backing for IOOS, this may improve in coming years.

\subsubsection{Integrated Marine Observing System (IMOS)}

In recent years, Australia has commenced the establishment of marine research-infrastructure around the country to systematically service Australia's significant requirements and responsibilities for one of the largest marine jurisdictions of any nation on earth. At over 14 million $\mathrm{km}^{2}$, Australia's Exclusive Economic Zone (EEZ) is nearly twice the surface area of the Australian continent. It extends from the tropics to Antarctic waters and much of it is unexplored.

The surrounding Pacific, Southern and Indian Oceans strongly affect the continental climate-system at all time scales, from seasons to decades. The major ocean currents on its eastern, western, northern and southern boundaries, (best known of these being the East Australian Current and the Leeuwin Current in the west), affect regional climatic conditions and marine ecosystems. There is evidence that these currents are 
changing on decadal time scales and have already impacted marine ecosystems, but the data are sparse and the currents and ecosystems have not been monitored in a systematic way. Management of climate impacts and sustainable use of the marine environment are major concerns in Australia, providing the rationale for large investments in the infrastructure to support relevant research. A challenge for IMOS (Integrated Marine Observing System) (http://www.imos.org.au/) from the beginning was to develop a broad consensus in the marine research community on a goal, national in scope, that would provide the basis for a national approach to marine observing. At the highest level that goal is to observe and support research on the impact of major boundary currents and regional ocean circulation on marine ecosystems and terrestrial climate.

IMOS was established as part of the Australian Government's National Collaborative Research Infrastructure Strategy $\quad$ (NCRIS) (http://ncris.innovation.gov.au). The NCRIS Roadmap outlines Australia's research infrastructure needs over the next five to 10 years. In launching the Roadmap the Minister for Innovation, Industry, Science and Research, the Hon Kim Carr, said, "The Government's objective is to work with universities, government organizations, non-profit research bodies and the private sector to invest in those research facilities that achieve the greatest return for Australia." Marine research was selected as an area for investment, in part because the marine research community expressed through a facilitation-process a strong consensus on the infrastructure that is required. The facilitation process culminated in an investment plan submitted to NCRIS.

After selection as one of the NCRIS programs (called capabilities) a governance structure was established to ensure that IMOS would take a national approach to marine observing. The IMOS Office established at the University of Tasmania is the body primarily responsible for coordination and management of the endeavor, which is implemented widely around the country. An Advisory Board with an independent Chair advises the Office on strategic approaches and approves the Annual Business Plans and Annual Progress Reports before they are sent to NCRIS. The Board members are appointed for outstanding abilities to guide the program and are senior leaders in the marine field able to take a broad, national perspective on IMOS development. The members serve in their own right and do not represent an organization. The IMOS Office also receives advice from a Scientific Steering Committee representing regional interests (Nodes (Network Operations, Design $\&$ Engineering Services) around the country. Each Node has about 50 members and they carry out their business guided by Terms of Reference, which are the same for each Node. The role of Nodes in IMOS is to provide the scientific rationale for the observing system and to promote the use of IMOS data for research. Each Node has prepared a Science and Implementation Plan calling for a specific set of observations.

The IMOS Office received an allocation of \$A50M from NCRIS for the period from 2007 to 2011 through a contractual agreement incorporating a Project Plan. The NCRIS funding was allocated to 10 Operators (Universities, State and Commonwealth agencies) to establish national Facilities through subcontracts. The Facilities are organized around building capability for deployment of specific sensors, for example a Facility for Argo floats, one for HF (High Frequency) coastal radar, etc. Co-investments from the Operators (largely in kind) nearly matched NCRIS funding making approximately $\$$ A94M available to develop the initial stage of IMOS. The Facilities deploy the observing infrastructure required by the Nodes.

The NCRIS funding and associated governance structure has provided a system comprised of a distributed set of oceanographic instruments, which provide streams of in situ data resulting in information services, which contribute to meeting the needs of marine research in Australia's open ocean and coastal waters. Ultimately, the streams of data are the infrastructure that has to be delivered to NCRIS. An additional \$A52M of federal funds were secured in 2009, as part of the Education Investment Fund (EIF) Super Science Initiative to enhance the existing observing system out to June 2013, and extend into Northern Australian and Southern Ocean waters. Such an investment is testament to the early success of IMOS.

The in situ data, when combined with satellite data, enables the modeling required to explain the role of the oceans in seasonal prediction and climate change. Sustaining the project will allow identification and management of climate change in the coastal marine environment. It will also provide an observational nexus to better understand and predict the fundamental connections between coastal biological processes and regional/oceanic phenomena that influence biodiversity. As an NCRIS project IMOS was primarily designed to support research; however, the data streams are also critical for societal, environmental and economic applications. Some of these include: management of marine natural resources and their associated ecosystems, support and management of coastal and offshore industries, safety at sea, marine tourism and defense. IMOS data are being used to validate and improve Australia's operational model for marine forecasting and services, BLUElink (Ocean forecasting Australia) (http://www.bom.gov.au/bluelink/).

IMOS, at the present time, has five regional Nodes covering the Great Barrier Reef, New South Wales (southeastern Australia), Southern Australia, Western Australia and the Bluewater and Climate Node. 
The observing Facilities include three for Bluewater and climate observations (Argo Australia, Enhanced Measurements from Ships of Opportunity and Southern Ocean Time Series), three facilities for coastal currents and water properties (Moorings, Ocean Gliders and HF Coastal Radar) and three for coastal ecosystems (Acoustic Tagging and Tracking, Autonomous Underwater Vehicle and a biophysical sensor network on the Great Barrier Reef). Two IMOS facilities are concerned with data management, one to assemble remote sensing data from satellites and a second to provide access to all IMOS data, and data services to all users.

\subsection{Data Management}

Below, the data management practices of each alliance are briefly reviewed. A community white paper dedicated to the topic of GOOS data management approaches by de La Beaujardière et al. [8] provides a more detailed discussion.

\subsubsection{EuroGOOS Data Management}

At present, the European ROOSs have different solutions for data management, which means that there is no single European system. The EuroGOOS Data Management and Exchange Working Group takes a long term perspective on European data management and puts forward recommendations for building on and synthesising the existing systems (e.g. SEADATANET (http://www.seadatanet.org)). The long term view for European marine data integration and management has recently been laid out by a joint EuroGOOS / ESF (European Science Foundation) activity described in the vision document for EDMODNET (European Marine Observation and Data Network, http://www.esf.org).

\subsubsection{IOOS-DMAC}

The nationally scoped IOOS [5] is a system-of-systems concept that leverages the Nation's existing capacity in ocean observations, modelling, and analysis-coupled via a Data Management and Communications (DMAC) component. Though not formally adopted, the early vision of DMAC was of a distributed system that effectively but robustly links federal and regional data providers into a seamless information system [7]. Several conceptual designs developed under contract to NOAA suggested that the system be centered around a series of data nodes (also called hubs or data centers), each of which would have either an agency, geographic or topical focus. Assuming each regional association acted as a data node, there would be roughly 1-2 dozen nodes in the national network. Critical to the distributed system concept is adoption of a standards process to ensure interoperability of all information contributors. An IOOS standards process was initiated in 2008 to formalize standards approval.
Technically, IOOS aims to harmonize the ways in which customers find, obtain and use information about the ocean. Data providers have traditionally offered data in disparate ways that make it difficult for users to gather a unified view of the seas from several sources. IOOS data providers will augment or replace existing access services and formats with standardized ones. The standards to be used are open, non-proprietary, vendorneutral standards adopted by GEOSS and developed by bodies such as the Open Geospatial Consortium (OGC) and the International Organization for Standardization (ISO). Providers will register their new services with an IOOS catalog to enable customers to find available data. Customers include decision-makers using highly specialized analysis tools, or simulation models that produce forecasts of weather or ocean conditions, or the general public via common web and desktop software. The complete IOOS DMAC will include additional services such as data visualization and format conversion.

In 2006, NOAA proposed building an initial operating capability, a data integration framework (DIF), for IOOS DMAC focusing on a limited subset of five ocean variables: temperature, salinity, sea level, currents, and ocean color (the list has since been augmented to include wind and wave measurements.) It has been noted that IOOS is a system comprised of many partner systems, each collecting data for specific purposes, and often in formats that differ from one another. As a result, data from each of these systems, even if the same variable, is often not compatible and cannot be used together without significant transformation by the user. The basic premise of the DIF is to identify a common set of community-based data standards and services that can be implemented at participating data providers enabling data from these distributed and disparate sources to be interoperable with one another. Additionally, NOAA IOOS is working with partners ("customers") from four decision-support areas of national importance to test and evaluate the value of the availability of this integrated data. These are: Hurricane Intensity forecasting, Coastal Inundation modelling, Harmful Algal Bloom forecasting, and Integrated Ecosystem Assessments. Details about this effort, including the DIF standards and services adopted can be found at http://ioos.noaa.gov/dif/. In partnership with the IOOS office, an initial set of three NOAA data providers have adopted and implemented these standardized data access services and formats, and the customers are now beginning to use data from the services in their products. This initial development project includes a plan to test and evaluate the resulting product enhancements and the standards and services used by all components of IOOS (federal and nonfederal). Favorable evaluation will lead to expansion of the framework to encompass additional ocean variables, 
data providers, customers, and data management functionalities, and will inform the decision regarding architecture and technologies for full operating capability and application to a National DMAC capability.

\subsubsection{IMOS Information management/services}

Management of the IMOS data streams occurs in two of the IMOS facilities: one to assemble remote sensing data from satellites and generate geo-referenced derived data products at full resolution (the Satellite Remote Sensing (SRS) service), and the second, the eMarine Information Infrastructure (eMII) project to assemble all IMOS data streams and products in a discoverable, interoperable and accessible framework. Marine data and information are the main products of IMOS and data management is therefore a central element to the project's success. eMII provides a single integrative framework for data access by scientists, managers and the public. The initial strategy has focused on defining specific data streams and developing end-to-end protocols, standards and systems to join the output from the observing Facilities into a unified data storage and access framework. A set of data products covering regional and national scales will ultimately be developed, as well as tools that facilitate the integration and analysis of data.

\subsubsection{IMOS data streams can be categorized in four} ways:

1) gridded data from satellites and HF radar systems;

2) time series data from moorings, Argo floats, gliders and ships of opportunity;

3) image data from Autonomous Underwater Vehicles;

4) biological data from continuous plankton recorders and acoustic tagging.

1) and 2) provide real-time and delayed-mode data sets whereas 3) and 4) are delayed mode delivery only.

The IMOS data management infrastructure employs Open Geospatial Consortium (OGC) standards wherever possible. The parallel structure with IOOS/DMAC opens a door to further integration. Additional storage formats and database protocols (e.g. WOCE exchange format, oracle) accommodate the data sets not readily converted to netCDF (network Common Data Form).

The primary data storage in eMII is a distributed network of OPeNDAP/THREDDS (Open-source Project for a Network Data Access Protocol/Thematic Real-time Environmental Distributed Data Services) servers around Australia, hosted by the Australian Research Collaboration Service (ARCS) 'Data Fabric' and utilizing the high-speed (10Gbit) dark fibre backbone of the Australian Academic and Research Network (AARNET). This complements the regional nodal structure of IMOS and allows rapid access to data by the local research community. Each local server also supports the GeoNetwork catalog (the Metadata Entry and Search Tool, MEST) with, wherever possible, automatic harvesting of metadata from the OPeNDAP/THREDDS system. An IMOS netCDF standard ensures that all necessary metadata complying with ISO 19115 can be automatically extracted from the netCDF files. Automation of metadata creation from non-netCDF datasets is also being investigated. A master GeoNetwork catalog at the University of Tasmania routinely harvests new metadata records from the regional catalogs to maintain a central registry.

DataTurbine streaming middleware provides a real-time view of IMOS time series data collected within the preceding month or two. A portal (http://imos.aodn.org.au) acts as a 'shop-window' to view IMOS data and as a data search engine utilising the GeoNetwork catalog tool. At present three 'views' of IMOS data are available: the real-time view through DataTurbine; a 'Facilities' view whereby all data from an IMOS facility, e.g. gliders, can be explored; and a 'Node' view whereby all data within an IMOS regional node, e.g. Southern Australia, can be explored.

Through the portal the GeoNetwork MEST search engine allows simple and complex data searches, both of IMOS data and other national and international datasets.

Accompanying the different views of IMOS data, a 'software toolbox' is under development containing a wide range of programs and scripts for access, visualisation, analysis and integration of IMOS data.

All IMOS data is freely available without constraints and is obtainable through a simple self registration process. As the IMOS program gains momentum the concept of data sharing and its value is spreading across Australia, a philosophy strongly endorsed by the Australian Federal Government's Super Science Initiative. Many universities and agencies are offering hitherto unavailable data streams for management and integration into IMOS. The long-term view of the data management infrastructure developed for IMOS is that it will become the infrastructure of the Australian Oceans Data Network (AODN), which is expected to encompass the full Australian marine community.

\section{ANALYSIS AND SUGGESTED WAY FORWARD}

The background material conveys the variety of audiences being targeted by regional programs and the variety of approaches taken in organizing those involved in ocean observing activities.

There are a number of similarities between the regional programs examined. The societal and environmental drivers for each system share commonalities. To support 
these objectives, each strives to organize activities on a continental scale, achieved through regional collaboration with some overarching coordination. They each envision connections to larger scale (global) and more holistic (earth observing) environmental networks, to maximize the utility of the information collected within and outside the regional system. The organizational structure used in each system is distributed, taking advantage of existing expertise. Additionally there are similarities in approaches and standards used for data management. All of these attributes are desirable and foster the communications and collaboration necessary to establish such large-scale integration of effort.

There are also noticeable differences between the regional programs. Both EuroGOOS and IOOS have been in development for more than a decade, and grew from modest starts through a growing network of interactions among interested parties. Remarkably, neither has yet achieved a stable formal status though both are expected to do so in the coming 1-2 years. Their relatively long history has helped foster awareness and support for the programs from a broad constituency, which is vital to their political success. In contrast, IMOS (Integrated Marine Observing System) is a new creation, structured to fit within a specific governmental construct, and fairly narrowly focused on establishing a national research endeavour. Unlike EuroGOOS and IOOS which most strongly promote the operational nature of the observing system and the services it can support, IMOS is being initiated as a research program that is anticipated to transition into a more operational program as it matures. This approach has the distinct advantage of engaging the research community en masse, which has at times been a challenge for EuroGOOS and IOOS. A possible disadvantage may be a lack of engagement of more operational components at an early stage of development (both governmental and private) and hence a lack of prioritization of their specific needs. A conscious effort to reach out to these communities will help alleviate this possible concern.

Related to the differing histories of the programs, each targets different audiences and has engaged somewhat different communities as participants. EuroGOOS, as an international effort, has most directly addressed the needs of national oceanographic and environmental agencies in its member states, and the majority of its participants come from these agencies. In contrast, IOOS, as a national program, has required that the federal agencies work together and its regional components engage a cross-section of participants, including state agency, academic and private interests. While this breadth of interests is laudable it can make prioritization of effort during times of scarce resources especially challenging, and as yet, no clear mechanism to adjudicate among all parties has been established. IMOS has chosen to initially limit its audience and participants to the research community, and requires that all investments support a national approach to ocean observing. Ideally, this is achieved by consensus in the community. There are merits and drawbacks to each approach.

As GOOS moves into its third decade, the need for compatibility among its many parts will become increasingly important. We encourage four activities to foster better communications and convergent evolution of design:

1. Assist in the documentation and sharing of governance frameworks being established to provide models for those just beginning these activities and to better inform those involved of their neighbors' governance structure. It will take time to align the presentation formats and there will be a need for a form of centralized access to the documentation and, therefore, some centralized support would be desirable. This support would logically come from GOOS

2. Promote planning and pilot projects that require cross-regional collaboration in providing specific services as a way to foster exchange of ideas and testing of compatibility between regions of the globe. EuroGOOS has championed this approach for a number of years and can provide examples of the approach. A simple initial approach would be to expand one or more of the EuroGOOS initiatives listed above to a multi-regional or global scale.

3. Nations around the Indian Ocean in particular would benefit from a cross-regional pilot project. Although lacking resources to develop observing systems and marine services, they have made some progress in initiating several national projects (see the OceanObs paper by Masumoto et al. for a summary.)

4. Initiate inter-regional and global data exchange pilot projects to test and harmonize data management practices as widely as possible at an early stage of development. A GOOS-hosted, limited-scope, multiregional project, which utilizes information from both global and regional programs is needed that will focus attention on adoption of international standards and protocols for data exchange. Early and frequent exercises to ensure global coordination are needed to avoid the costs of re-tooling at a later date.

A forward vision of regional and coastal ocean observing has to address the gap between the Global and Coastal modules of GOOS. We have not analyzed why this gap exists, but it seems evident that the two modules have emerged from quite distinct academic research disciplines and have somewhat different cultures. The rapid advances being made now in ocean and climate modelling, down scaling, ocean-reanalysis and operational marine services provides an opportunity 
and a framework for integration of the Global and Coastal modules.

We believe these initial steps will raise awareness of alternative approaches and speed the selection of best practices for broad implementation.

\section{REFERENCES}

1. Briscoe, M. G., D. L. Martin and T. C. Malone, 2008. Evolution of regional efforts in international GOOS and U.S. IOOS, MTS Journal, 42(3), 4-9.

2. NORLC, 1999a: Toward a U.S. Plan for an Integrated, Sustained Ocean Observing System. http://www.nopp.org/resources/nopp/towardintegrated.p df, $85 \mathrm{pp}$.

3. NORLC, 1999b: An Integrated Ocean Observing System: A Strategy for Implementing the First Steps of a U.S. Plan. http://www.nopp.org/iDuneDownload.dll?GetFile?AppI $\mathrm{d}=141 \&$ FileID=235157\&Anchor=\&ext=.pdf, 39pp.

4. Ocean.US 2002. An integrated and sustained ocean observing system (IOOS) for the United States: design and implementation. Ocean.US, Report No. 2,

Arlington, VA.

http:///www.ocean.us/documents/docs/FINAL-ImpPlanNORLC.pdf, $21 \mathrm{pp}$.

5. Ocean.US 2006a. The first US integrated ocean observing system (IOOS) development plan. Ocean.US, Report No. 9, Arlington, VA.

http:///www.ocean.us/documents/docs/IOOSDevPl an_low-res.pdf, 104 pp.

6. Ocean.US 2006b. U.S. GOOS national report - US national implementation and planning activities 2005 highlights. Ocean.US, Report No. 14, Arlington, VA.

http:///www.ocean.us/documents/docs/GOOS_4_lowres.pdf, $32 \mathrm{pp}$.

7. Ocean.US 2007. Proceedings, second IOOS implementation conference: multi-hazard forecasting. Ocean.US, Report No. 10, Arlington, VA.

http:///www.ocean.us/documents/docs/2ndIOOS_FINA L low-res.pdf, 58 pp.

8. de La Beaujardière, J. \& Co-Authors (2010). "Ocean and Coastal Data Management" in these proceedings (Vol. 2), doi:10.5270/OceanObs09.cwp.22 